\title{
High expression of IncRNA-SNHG7 is associated with poor prognosis in hepatocellular carcinoma
}

\author{
AN SHEN $^{1}$, JINPING MA $^{2}$, XIAOLIN HU $^{3}$ and XINJIANG CUI ${ }^{4}$ \\ Departments of ${ }^{1}$ Hepatological Surgery and ${ }^{2}$ Obstetrics, Affiliated Hospital of Weifang Medical University, \\ Weifang, Shandong 261041; ${ }^{3}$ Department of General Surgery, Shouguang People's Hospital, \\ Shouguang, Shandong 262700; ${ }^{4}$ Department of Interventional Oncology, Affiliated Hospital \\ of Weifang Medical University, Weifang, Shandong 261041, P.R. China
}

Received October 17, 2019; Accepted December 13, 2019

DOI: $10.3892 / \mathrm{ol} .2020 .11490$

\begin{abstract}
Expression of long non-coding RNA SNHG7 (lncRNA-SNHG7) and its clinical significance in hepatocellular carcinoma (HCC) were explored. Quantitative real-time polymerase chain reaction (qRT-PCR) was used to detect the expression level of lncRNA-SNHG7 in cancer tissues. KaplanMeier curves and multivariate Cox proportional models were used to study the impact on clinical outcome. Expression of lncRNA-SNHG7 was much higher in cancer tissues than that in para-cancer tissues. The lncRNA-SNHG7 expression was correlated with tumor number, lymph node metastasis and clinical stage $(\mathrm{P}<0.05)$. In addition, HCC patients with higher lncRNA-SNHG7 expression had significantly poorer progression-free survival time and overall survival time $(\mathrm{P}<0.001)$. Both univariate analysis and multivariate analysis indicated that high expression of 1ncRNA-SNHG7 was an independent predictor of poor prognosis in HCC. LncRNA-SNHG7 might contribute to the development of $\mathrm{HCC}$ and serve as a clinical biomarker and a therapeutic target for HCC patients.
\end{abstract}

\section{Introduction}

Hepatocellular carcinoma (HCC) is a common malignant tumor worldwide, with increasing incidence rate and mortality rate. More than 700,000 new cases emerge each year, and its mortality rate ranks third among malignant tumors $(1,2)$. There is often no specific clinical manifestation in the early stage, so the vast majority of HCC patients tend to be diagnosed in the late stage. Once distant metastasis occurs, its prognosis deteriorates significantly, and the survival rate after surgery

Correspondence to: Dr Xinjiang Cui, Department of Interventional Oncology, Affiliated Hospital of Weifang Medical University, 2428 Yuhe Road, Kuiwen, Weifang, Shandong 261041, P.R. China E-mail: xiannei19860913@163.com

Key words: hepatocellular carcinoma, long non-coding RNA SNHG7, progression-free survival, overall survival will sharply decline. To date, metastasis and recurrence have constituted major causes of death for HCC patients $(3,4)$.

Long non-coding ribonucleic acids (lncRNAs) are RNAs that cannot encode proteins, with approximately 200 nucleotides in length $(5,6)$. Originally considered as 'background noise' or 'junk DNA', lncRNAs were deemed as useless RNAs, which exert no influence on the transcription and translation of proteins $(7,8)$. However, recent years have seen increasing number of studies that have pointed out that lncRNAs play an important role in the biological process as necessary regulators to promote or inhibit the growth of transcribed tumor cells or to spur tumor metastasis (9-12). Long non-coding RNA SNHG7 (lncRNA-SNHG7), which is 2176 bp-long and located on chromosome 9q34.3, is a recognized bidirectional lncRNA. It was reported that it could guide snoRNA to undergo posttranslational modification (13). Furthermore, it was a ribosomal RNA involved in modifying the growth and proliferation of cells. Abnormal expression of snoRNA is a potential factor for carcinogenesis. It was reported that lncRNA-SNHG7 was able to affect the regulation of proliferation, metastasis and invasion and suppress apoptosis of malignant tumor cells including lung cancer (14), pancreatic cancer (15), esophageal cancer (16) and gastric cancer (17). However, its role in HCC has remained unclear.

The present study explored the expression of IncRNASNHG7 and its clinical significance in HCC with the aim of contributing to the clarification of the biological mechanism of the tumor as well as providing valuable targets for corresponding diagnosis and treatment.

\section{Patients and methods}

Collection of tissue specimens. This study was approved by the Ethics Committee of Affiliated Hospital of Weifang Medical University (Weifang, China). Signed informed consents were obtained from all participants before entry to the study. The HCC tissues and para-carcinoma tissues $(>5 \mathrm{~cm}$ away from cancer tissues) were harvested from 100 patients undergoing radical or palliative resection from April 2016 to December 2018, and the personal information and detailed clinical data of patients, including patient's sex, age, AFP levels, HBsAg levels, tumor size, tumor number, lymph node 


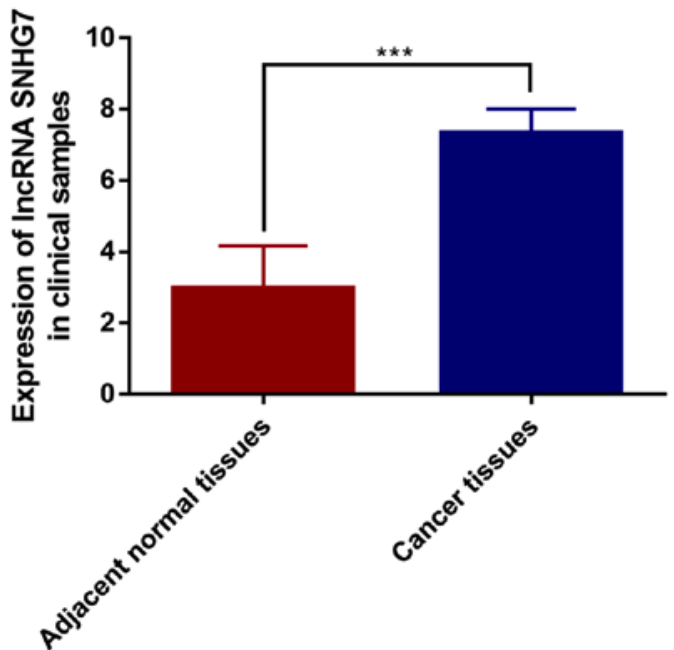

Figure 1. Expression level of lncRNA SNHG7 was evaluated in cancer tissues and para-cancer tissues by qRT-PCR. IncRNA-SNHG7, long noncoding RNA SNHG7; qRT-PCR, quantitative real-time polymerase chain reaction $\left({ }^{* * * *} \mathrm{P}<0.001\right)$.

metastasis and clinical stage were collected intact. Patients treated by radiotherapy and chemotherapy were excluded. The tissues were pathologically diagnosed with HCC, and the freshly-resected specimens were immediately cryopreserved in liquid nitrogen until quantitative real-time polymerase chain reaction (qRT-PCR) was performed.

QRT-PCR analysis. RNAs in primary HCC tissues and normal adjacent tissues were extracted according to the operation steps given in the specification of TRIzol reagent (Invitrogen; Thermo Fisher Scientific, Inc.), and lncRNA-SNHG7 gene was subjected to PCR based on the specification of reverse transcription kit. The conditions of PCR were as follows: pre-denaturation at $95^{\circ} \mathrm{C}$ for $10 \mathrm{~min}$, followed by a total of 40 cycles for $15 \mathrm{sec}$ at $95^{\circ} \mathrm{C}, 1 \mathrm{~min}$ at $60^{\circ} \mathrm{C}$ and $30 \mathrm{sec}$ at $72^{\circ} \mathrm{C}$. The relative expression level of lncRNA-SNHG7 was calculated by $2^{-\Delta \Delta C t}$ method. Primer sequences used in the study are shown below: SNHG7 F: 5'-GTGTGTCCCTTGGTGGAGAG-3'; R: TCCCAGAT ACCAGCGAAGGA-3'. GAPDH F: 5'-AGAAGGCTGGGG CTCATTTG-3'; R: 5'-AGGGGCCATCCACAGTCTTC-3'.

Patient follow-up. The follow-up was conducted by phone or outpatient visit to record the survival of patients. The deadline for follow-up was 7 years. The total survival period was from the date of onset to the date of the final follow-up or death, in months.

Statistical analysis. Statistical Product and Service Solutions (SPSS) 19.0 software (IBM Corp.) was selected for the processing of result data, and measurement data were expressed as mean \pm standard deviation (mean $\pm \mathrm{SD}$ ) or median. Paired t-test was used to compare the expression level of IncRNA-SNHG7 in primary HCC tissues and normal adjacent tissues. Group $\chi^{2}$ test was adopted to analyze the associations of the expression of lncRNA-SNHG7 in HCC tissues with the clinicopathological features of patients. Overall survival (OS) and progression-free survival (PFS) of the patients was evaluated via Kaplan-Meier survival
Table I. lncRNA-SNHG7 expression and clinical features of patients with HCC.

\begin{tabular}{|c|c|c|c|c|}
\hline \multirow[b]{2}{*}{ Features } & \multirow[b]{2}{*}{ No. } & \multicolumn{2}{|c|}{$\begin{array}{l}\text { lncRNA- } \\
\text { SNHG7 }\end{array}$} & \multirow[b]{2}{*}{ P-value } \\
\hline & & High & Low & \\
\hline No. & 100 & 53 & 47 & \\
\hline Sex & & & & 0.930 \\
\hline Male & 66 & 34 & 32 & \\
\hline Female & 34 & 19 & 15 & \\
\hline Age (years) & & & & 0.815 \\
\hline$<60$ & 43 & 23 & 20 & \\
\hline$\geq 60$ & 57 & 30 & 27 & \\
\hline AFP & & & & 0.894 \\
\hline$<20$ & 28 & 16 & 12 & \\
\hline$\geq 20$ & 72 & 37 & 35 & \\
\hline HBsAg & & & & 0.922 \\
\hline Positive & 60 & 31 & 29 & \\
\hline Negative & 40 & 22 & 18 & \\
\hline Tumor size (cm) & & & & 0.886 \\
\hline$<5$ & 39 & 20 & 19 & \\
\hline$\geq 5$ & 61 & 33 & 28 & \\
\hline Tumor number & & & & 0.001 \\
\hline Solitary & 69 & 25 & 44 & \\
\hline Multiple & 31 & 28 & 3 & \\
\hline Lymph node metastasis & & & & 0.008 \\
\hline Absence & 62 & 24 & 38 & \\
\hline Presence & 38 & 29 & 9 & \\
\hline Clinical stage & & & & 0.012 \\
\hline $\mathrm{I}+\mathrm{II}$ & 67 & 30 & 37 & \\
\hline $\mathrm{III}+\mathrm{IV}$ & 33 & 23 & 10 & \\
\hline
\end{tabular}

lncRNA-SNHG7, long non-coding RNA SNHG7; HCC, hepatocellular carcinoma.

analysis and the intergroup differences were analyzed by log rank test. Cox proportional hazard regression model was, respectively, chosen for single factor analysis and multiple factor analysis of survival. $\mathrm{P}<0.05$ was considered to indicate a statistically significant difference.

\section{Results}

Expression of lncRNA-SNHG7 in HCC tissues. Expression of lncRNA-SNHG7 in 100 pairs of cancer tissues and para-normal tissues was detected. The results showed that the expression of lncRNA-SNHG7 in HCC tissues was much higher than that of para-normal tissues, the difference was statistically significant $(\mathrm{P}<0.001)$ (Fig. 1). These results are consistent with IncRNASNHG7 described in literature.

The HCC samples were divided into SNHG7-high expression group $(n=53)$ and SNHG7-low expression group $(n=47)$ based on the mean expression level of lncRNA-SNHG7 from 
Table II. Univariate and multivariate analyses of postoperative prognosis in patients with gastric cancer.

\begin{tabular}{lccccc}
\hline & \multicolumn{2}{c}{ Univariate analysis } & & \multicolumn{2}{c}{ Multivariate analysis } \\
\cline { 2 - 3 } Features & Hazard ratio/CI (95\%) & P-value & & Hazard ratio/CI (95\%) & P-value \\
\hline Sex & $0.983 / 0.754-1.248$ & 0.868 & & \\
Age & $1.103 / 0.813-1.199$ & 0.913 & & \\
Tumor size & $1.627 / 0.917-2.425$ & 0.071 & & \\
Tumor position & $0.955 / 0.600-1.136$ & 0.492 & & \\
Tumor differentiation & $2.120 / 1.138-3.869$ & 0.033 & & $2.006 / 1.091-3.106$ & \\
T stage & $1.455 / 0.920-2.861$ & 0.195 & & & 0.030 \\
N stage & $3.374 / 1.821-5.429$ & 0.007 & & $3.173 / 1.720-4.851$ & 0.027 \\
M stage & $3.987 / 2.349-4.780$ & 0.012 & & $3.294 / 2.322-4.234$ & 0.008 \\
TNM stage & $4.102 / 3.076-5.371$ & 0.003 & & $3.722 / 2.926-5.110$ & 0.015 \\
lncRNACADM1-AS1 expression level & $2.994 / 1.670-4.051$ & 0.009 & & $2.584 / 1.621-3.880$ & 0.015 \\
\hline
\end{tabular}

TNM, tumor-node-metastasis; lncRNA, long non-coding ribonucleic acid.

qRT-PCR analysis. The relationship between lncRNA-SNHG7 expression and clinicopathological features of patients was further analyzed, and it was found that there was no statistical difference between lncRNA-SNHG7 expression and patient's sex, age, AFP levels, HBsAg levels or tumor size ( $\mathrm{P}>0.05)$. However, statistical difference was found between IncRNASNHG7 expression and tumor number, lymph node metastasis and clinical stage (Table I).

Effect of lncRNA-SNHG7 on the prognosis of patients with $H C C$. Correlation between 1ncRNA-SNHG7 expression and survival time of patients was evaluated using the Kaplan-Meier method. The results revealed that the patients with high expression of lncRNA-SNHG7 had worse progression-free survival (PFS) and overall survival (OS) time compared to SNHG7-low expression patients. The results were statistically significant $(\mathrm{P}<0.001)$ (Fig. 2), indicating high expression of lncRNASNHG7 in HCC patients predicted poor prognosis.

Univariate and multivariate analyses of IncRNA-SNHG7 expression and HCC clinicopathological data. Univariate Cox proportional hazards regression model analysis was used to analyze pathological parameters of HCC. The results revealed that tumor number, lymph node metastasis, clinical stage and lncRNA-SNHG7 expression level were statistically significant risk factors.

The above four factors with significant influence were incorporated into the multivariate Cox proportional hazard model for further analysis (Table II). Similarly to tumor number, lymph node metastasis and clinical stage. Expression of lncRNA-SNHG7 was an independent prognosis index of patients with $\mathrm{HCC}(\mathrm{P}<0.05)$. These findings suggested that lncRNA-SNHG7 might play an important role in the progression of HCC.

\section{Discussion}

$\mathrm{HCC}$ is characterized by insidious onset and rapid progress. The vast majority of patients are diagnosed in the middle
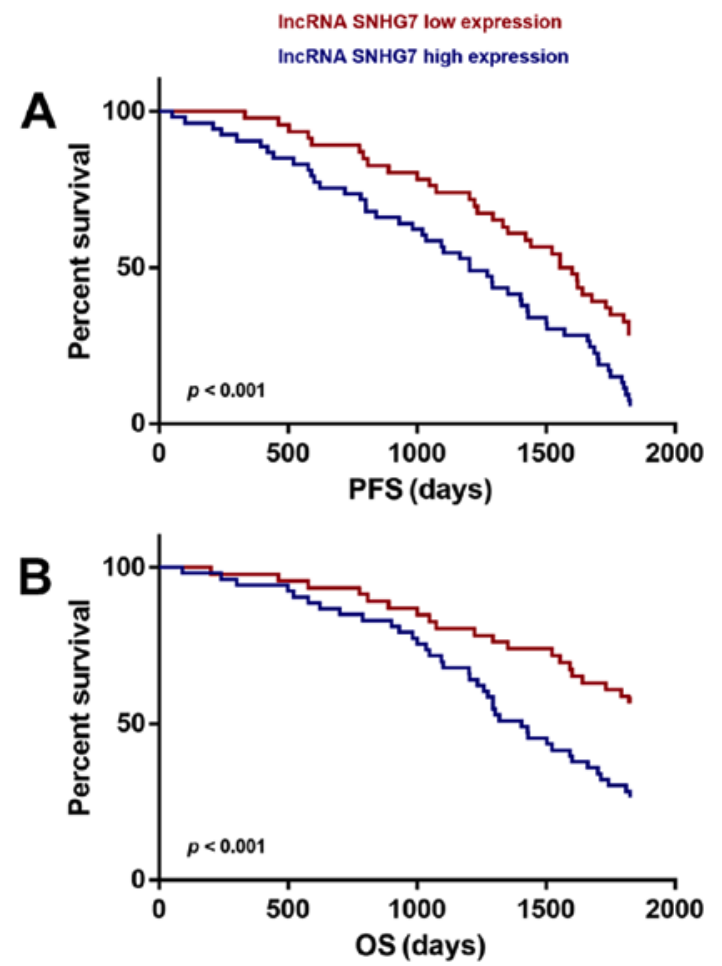

Figure 2. Relationship of 1ncRNA SNHG7 expression with progression-free survival (PFS) (A) and overall survival (OS) (B) of HCC patients. IncRNASNHG7, long non-coding RNA SNHG7; HCC, hepatocellular carcinoma.

and late stages, thus missing the best time for surgery. Even with radical resection, the postoperative recurrence and metastasis still significantly impair the long-term survival of patients $(18,19)$. In order to realize early detection of HCC and improve the prognosis of patients, the cellular and molecular biological mechanisms leading to $\mathrm{HCC}$ invasion and metastasis have attracted extensive attention and study.

Sequencing of the human genome indicated that proteinencoding genes account for $<3 \%$ of all sequences in the human genome, and $>80 \%$ of the sequences are frequently 
transcribed into RNAs without protein-encoding functions, called non-coding RNAs. They are a complex network that regulates gene expression and a key to the regulation of many essential biological functions of tumor cells $(10,20,21)$. They are categorized into short non-coding RNAs and lncRNAs based on different sequence lengths (12). IncRNAs cannot be translated into proteins, but they could affect gene transcription, protein modification after translation and epigenetic regulation $(22,23)$. A growing number of studies have shown that lncRNAs are abnormally expressed in various types of tumors, which imply their potential value in the early diagnosis of certain tumors and were considered as markers for early diagnosis. IncRNA-JADE was found with a key functional link that connects the DNA damage response (DDR) to histone $\mathrm{H} 4$ acetylation, and that dysregulation of lncRNAJADE might contribute to breast tumorigenesis (24). The positive lncRNA-HOTAIR/HER2 correlation was associated with gastric cancer development. High expression of HOTAIR was associated with shorter overall survival of gastric cancer patients (25). LncRNA MALAT-1 was positively related to Bcl-2 expression in lung cancer and weak MALAT-1 expression patients with resected lung cancer demonstrated adverse prognosis (26).

Various studies have pointed out that lncRNA-SNHG7 is highly expressed in various malignant tumors and is a potential molecular marker. Qi et al (27) indicated that through miR-503/cyclin D1 pathway, IncRNA-SNHG7 promoted the development of prostate cancer. Colorectal cancer patients with high expression of lncRNA-SNHG7 had a significantly poor prognosis (28). Further results indicated that lncRNASNHG7 facilitated the proliferation and metastasis of tumor cells by regulating GALNT7 expression and PI3K/Akt/mTOR pathway. She et al (14) found that IncRNA-SNHG7 was highly expressed in lung cancer tissues, and the inhibition of lncRNASNHG7 expression could promote apoptosis of lung cancer cells. The role of lncRNA-SNHG7 in lung cancer was through the regulation of FAIM2 gene. The above studies indicated that lncRNA-SNHG7 might become a potential target for the treatment of tumors. However, so far, studies on the expression and biological functions of lncRNA-SNHG7 in tumor tissues are relatively few, and there are no relevant reports involving the field of HCC.

It was discovered in this study that the expression level of lncRNA-SNHG7 in HCC tissues was much higher than that in normal adjacent tissues, suggesting that the inhibition of lncRNA-SNHG7 expression might be related to the occurrence and procession of $\mathrm{HCC}$. The correlation of the expression of lncRNA-SNHG7 with clinicopathological features of HCC patients found that the high expression of lncRNA-SNHG7 in HCC tissues was related to tumor number, lymph node metastasis and clinical stage but not associated with patient's sex, age, AFP levels, HBsAg levels and tumor size.

Multidisciplinary team (MDT) diagnosis and treatment of HCC patients by various treatment methods has gradually become a characteristic trend of HCC treatment (29). In clinical practice, certain indicators that were capable of accurately predicting the prognosis would promote the identification of people with strong recurrence tendency and provide the corresponding basis for prevention and treatment, thus preventing recurrence as early as possible. This study analyzed the clinicopathological features of $100 \mathrm{HCC}$ patients, and revealed that high expression of lncRNA-SNHG7 in HCC patients indicated a poor prognosis. Moreover, the results of univariate analysis and multivariate analysis model showed that lncRNA-SNHG7 expression was an influential factor for the prognosis of HCC patients. Clinical detection of IncRNASNHG7 expression level might assist in making the prognosis of HCC patients.

In conclusion, IncRNA-SNHG7 might contribute to the development of $\mathrm{HCC}$ and serve as a clinical biomarker and a therapeutic target for $\mathrm{HCC}$ patients.

\section{Acknowledgements}

Not applicable.

\section{Funding}

No funding was received.

\section{Availability of data and materials}

All data generated or analyzed during this study are included in this published article.

\section{Authors' contributions}

AS and XC designed the study and performed the experiments, AS and JM collected the data, XC and XH analyzed the data, AS and XC prepared the manuscript. All authors read and approved the final manuscript.

\section{Ethics approval and consent to participate}

This study was approved by the Ethics Committee of Affiliated Hospital of Weifang Medical University (Weifang, China). Signed informed consents were obtained from the patients and/or the guardians.

\section{Patient consent for publications}

Not applicable.

\section{Competing interests}

The authors declare that they have no competing interests.

\section{References}

1. Siegel RL, Miller KD and Jemal A: Cancer statistics, 2018. CA Cancer J Clin 68: 7-30, 2018.

2. Braillon A: Hepatocellular carcinoma. Lancet 380: 469-471, 2012.

3. Lau WY and Lai EC: Hepatocellular carcinoma: Current management and recent advances. Hepatobiliary Pancreat Dis Int 7: 237-257, 2008

4. El-Serag HB and Rudolph KL: Hepatocellular carcinoma: Epidemiology and molecular carcinogenesis. Gastroenterology 132: 2557-2576, 2007.

5. Mercer TR, Dinger ME and Mattick JS: Long non-coding RNAs: Insights into functions. Nat Rev Genet 10: 155-159, 2009.

6. Wilusz JE, Sunwoo H and Spector DL: Long noncoding RNAs: Functional surprises from the RNA world. Genes Dev 23: 1494-1504, 2009. 
7. Kelley D and Rinn J: Transposable elements reveal a stem cellspecific class of long noncoding RNAs. Genome Biol 13: R107, 2012.

8. Bonasio R and Shiekhattar R: Regulation of transcription by long noncoding RNAs. Annu Rev Genet 48: 433-455, 2014

9. Gupta RA, Shah N, Wang KC, Kim J, Horlings HM, Wong DJ, Tsai MC, Hung T, Argani P, Rinn JL, et al: Long non-coding RNA HOTAIR reprograms chromatin state to promote cancer metastasis. Nature 464: 1071-1076, 2010.

10. Esteller M: Non-coding RNAs in human disease. Nat Rev Genet 12: 861-874, 2011.

11. Eddy SR: Non-coding RNA genes and the modern RNA world. Nat Rev Genet 2: 919-929, 2001.

12. Ørom UA, Derrien T, Beringer M, Gumireddy K, Gardini A, Bussotti G, Lai F, Zytnicki M, Notredame C, Huang Q, et al: Long noncoding RNAs with enhancer-like function in human cells. Cell 143: 46-58, 2010.

13. Chaudhry MA: Small nucleolar RNA host genes and long noncoding RNA responses in directly irradiated and bystander cells. Cancer Biother Radiopharm 29: 135-141, 2014.

14. She K, Huang J, Zhou H, Huang T, Chen G and He J: lncRNASNHG7 promotes the proliferation, migration and invasion and inhibits apoptosis of lung cancer cells by enhancing the FAIM2 expression. Oncol Rep 36: 2673-2680, 2016.

15. Cheng D, Fan J, Ma Y,Zhou Y, Qin K, Shi M and Yang J: LncRNA SNHG7 promotes pancreatic cancer proliferation through ID4 by sponging miR-342-3p. Cell Biosci 9: 28, 2019.

16. Xu LJ, Yu XJ, Wei B, Hui HX, Sun Y, Dai J and Chen XF LncRNA SNHG7 promotes the proliferation of esophageal cancer cells and inhibits its apoptosis. Eur Rev Med Pharmacol Sci 22: 2653-2661, 2018.

17. Wang MW, Liu J, Liu Q, Xu QH, Li TF, Jin S and Xia TS LncRNA SNHG7 promotes the proliferation and inhibits apoptosis of gastric cancer cells by repressing the P15 and P16 expression. Eur Rev Med Pharmacol Sci 21: 4613-4622, 2017.

18. Altekruse SF, McGlynn KA and Reichman ME: Hepatocellular carcinoma incidence, mortality, and survival trends in the United States from 1975 to 2005. J Clin Oncol 27: 1485-1491, 2009.

19. Chang CH, Chau GY, Lui WY, Tsay SH, King KL and Wu CW: Long-term results of hepatic resection for hepatocellular carcinoma originating from the noncirrhotic liver. Arch Surg 139: 320-326, 2004.
20. Stefani G and Slack FJ: Small non-coding RNAs in animal development. Nat Rev Mol Cell Biol 9: 219-230, 2008.

21. Griffiths-Jones S, Moxon S, Marshall M, Khanna A, Eddy SR and Bateman A: Rfam: Annotating non-coding RNAs in complete genomes. Nucleic Acids Res 33: D121-D124, 2005

22. Zhang Q and Jeang KT: Long non-coding RNAs (lncRNAs) and viral infections. Biomed Pharmacother 3: 34-42, 2013.

23. Carlevaro-Fita J, Rahim A, Guigó R, Vardy LA and Johnson R: Cytoplasmic long noncoding RNAs are frequently bound to and degraded at ribosomes in human cells. RNA 22: 867-882, 2016.

24. Wan G, Hu X, Liu Y, Han C, Sood AK, Calin GA, Zhang X and Lu X: A novel non-coding RNA IncRNA-JADE connects DNA damage signalling to histone $\mathrm{H} 4$ acetylation. EMBO J 32: 2833-2847, 2013.

25. Liu XH, Sun M, Nie FQ, Ge YB, Zhang EB, Yin DD, Kong R, $\mathrm{Xia} \mathrm{R}$, Lu KH, Li JH, et al: Lnc RNA HOTAIR functions as a competing endogenous RNA to regulate HER 2 expression by sponging miR-331-3p in gastric cancer. Mol Cancer 13: 92, 2014.

26. Schmidt LH, Görlich D, Spieker T, Rohde C, Schuler M, Mohr M, Humberg J, Sauer T, Thoenissen NH, Huge A, et al: Prognostic impact of Bcl-2 depends on tumor histology and expression of MALAT-1 lncRNA in non-small-cell lung cancer. J Thorac Oncol 9: 1294-1304, 2014.

27. Qi H, Wen B, Wu Q, Cheng W, Lou J, Wei J, Huang J, Yao X and Weng G: Long noncoding RNA SNHG7 accelerates prostate cancer proliferation and cycle progression through cyclin D1 by sponging miR-503. Biomed Pharmacother 102: 326-332, 2018.

28. Li Y, Zeng C, Hu J, Pan Y, Shan Y, Liu B and Jia L: Long non-coding RNA-SNHG7 acts as a target of miR-34a to increase GALNT7 level and regulate PI3K/Akt/mTOR pathway in colorectal cancer progression. J Hematol Oncol 11: 89, 2018.

29. Siddique O, Yoo ER, Perumpail RB, Perumpail BJ, Liu A, Cholankeril $\mathrm{G}$ and Ahmed A: The importance of a multidisciplinary approach to hepatocellular carcinoma. J Multidiscip Healthc 10: 95-100, 2017.

This work is licensed under a Creative Commons Attribution-NonCommercial-NoDerivatives 4.0 International (CC BY-NC-ND 4.0) License. 